\title{
Índice padronizado de precipitação aplicado às condições de seca no Estado do Espírito Santo
}

\author{
Gabriel C. Blain ${ }^{1}$, José R. M. Pezzopane'2, José E. M. Pezzopane 3 \& Robson Bonomo ${ }^{4}$
}

RESUMO

\begin{abstract}
0 Índice Padronizado de Precipitação (SPI) é um dos métodos mais utilizados para quantificação da seca. A fim de verificar a possibilidade de utilização do SPI no monitoramento das deficiências e excessos de precipitação na escala mensal, no Estado do Espírito Santo objetivou-se, neste trabalho, verificar o ajuste das séries temporais dessa variável meteorológica à distribuição gama em cinco localidades do Estado. Por meio dos testes de aderência Kolmogorov-Smirnov e qui-quadrado, as séries mensais de precipitação pluvial das localidades sob análise podem ser consideradas oriundas de uma população com distribuição gama incompleta, permitindo o uso do SPI no monitoramento das condições de seca meteorológica. Através de análises de autocorrelação e correlação-cruzada, observou-se que a principal característica das séries do SPI é sua grande variabilidade espaço-temporal, a qual indica que em uma mesma região meses extremamente secos podem ser precedidos e/ou seguidos de meses úmidos ou normais, e que distintos casos de seca podem ocorrer de forma aleatória, entre as localidades e em um mesmo período.
\end{abstract}

Palavras-chave: distribuição gama, teste de aderência, precipitação pluvial

\section{Standardized precipitation index applied to drought conditions of the State of Espírito Santo, Brazil}

\begin{abstract}
The Standardized Precipitation Index (SPI) is one of the most widely used methods for quantification of drought conditions. In order to apply the SPI model to the climate conditions of the State of Espírito Santo, Brazil, the study evaluated the adjustment of the monthly rainfall time series to the gamma distribution in five regions of this State. U sing the KolmogorovSmirnov test and the Chi-Square test, it was possible to verify that the monthly rainfall time series, used in this study, can be fitted to the gamma distribution. On this sense, the SPI model can be applied for monitoring the meteorological drought conditions in the State of Espírito Santo. U sing the autocorrelation function and the cross-correlation function, it was possible to verify that the main characteristic of the monthly SPI series is high temporal variability of its values. This indicates that, within a same region, extreme dry months may be preceded and/or followed by normal or wet months, and different drought condition can be observed, at the same period, among the five analyzed regions.
\end{abstract}

Key w ords: gamma distribution, goodness-of-fit test, rainfall time series

\footnotetext{
${ }^{1}$ IAC/CEB, Av. Barão de Itapura 1500, Guanabara, CP 28, CEP 13001-970, Campinas-SP. Fone: (19) 3242-5230. E-mail: gabriel@iac.sp.gov.br ${ }^{2}$ Embrapa Pecuária Sudeste, Rod. Washington Luis, Km 234, CEP 13563-776, São Carlos, SP. Fone: (16) 3411-5620. E-mail: jricardo@cppse.embrapa.br ${ }^{3}$ Centro de Ciências Agrárias/UFES, Alto Universitário, s/n 257, CP 16, Guararema, CEP 29500-000, Alegre, ES. Fone: (28) 3552-1565. E-mail: jemp@cca.ufes.br ${ }^{4}$ Centro Universitário Norte do Espírito Santo/UFES, Rua Humberto de Almeida Franklin 257, Bairro U niversitário, CEP 29933-480, São Matheus, ES. Fone: (27) 3763-8697. E-mail: robsonbonomo@ceunes.ufes.br
} 


\section{INTRODUÇÃO}

A seca é um fenômeno meteorológico complexo, com influências sociais frequentemente agravadas pela ação humana. A ocorrência dessa anomalia climática em várias regiões do globo terrestre evidencia a vulnerabilidade do homem a esse risco climático, demonstrando a necessidade de um entendimento melhor, de melhor previsão de sua ocorrência e do uso correto de medidas mitigatórias (Wilhite \& Glantz,1987).

Neste sentido, vários indicadores podem ser desenvolvidos para quantificar, padronizar e comparar a seca, em base temporal e espacial, dentre eles o Índice Padronizado de Precipitação (Standardized Precipitation Index, SPI), desenvolvido por Mckee et al. (1993) visto que se trata, atualmente, de um dos mais utilizados. O SPI quantifica o déficit ou o excesso de precipitação em diferentes escalas de tempo, característica que torna este índice um valioso método para todos os estudos de disponibilidade hídrica, sejam eles de curta ou de longa duração (Hayes et al., 1999). O primeiro passo para o cálculo do SPI é a determinação da probabilidade de distribuição de frequência de precipitação pluvial calculada por meio da distribuição gama incompleta. A função normal inversa (Gaussiana) é aplicada a esta probabilidade, cujo resultado é o SPI.

Do ponto de vista agrometeorológico, a precipitação pluvial é um dos elementos climáticos mais diretamente relacionados à produção agronômica sendo que seu caráter aleatório (variações e anomalias) dificulta a programação das atividades do setor agrícola (Melo Júnior et al., 2006); neste sentido, Vieira et al. (1994) e Silva et al. (2003) afirmam que a precipitação pluviométrica tem grande importância na caracterização do clima de uma região, interferindo nas alternâncias de rendimento das culturas e na construção civil; desta forma torna-se evidente a relevância de métodos que detectam e quantificam, de forma simples e rotineira, o início e a severidade de uma seca.

Do ponto de vista conceitual não há, na literatura, uma definição do fenômeno seca, que seja válida para todas as atividades humanas; assim, é comum a definição de tipos de seca: agrícola, meteorológica, hidrológica e socioeconômica. Apesar dessa dificuldade, e segundo Wilhite et al. (1987) todas as diferentes formas dessa anomalia climática são originárias de um déficit de precipitação que resulta em baixa disponibilidade hídrica para a atividade que a requer.

Para verificar, na escala mensal, a possibilidade de utilização do SPI no monitoramento das deficiências e excessos de precipitação no Estado do Espírito Santo propôs-se, no trabalho, verificar o ajuste das séries temporais dessa variável meteorológica à distribuição gama, em cinco localidades do Estado. Inferências sobre as formas e escalas das distribuições mensais de precipitação, análises de autocorrelação (persistência) e correlação cruzada dos valores do SPI, também foram realizadas.

\section{MATERIAL E MÉTODOS}

De acordo com a classificação de Köppen, o clima dos municípios de Alegre, Baixo Guandu, Ecoporanga e São Mateus, é do tipo Aw (Tropical com inverno seco) e o clima do município de Santa Tereza, localizado na região serrana do estado, é do tipo Subtropical Quente sem estação seca (Cfb). A Figura 1 demonstra a localização geográfica das estações. O período comum de 1977 a 2006 foi adotado em todas as localidades.

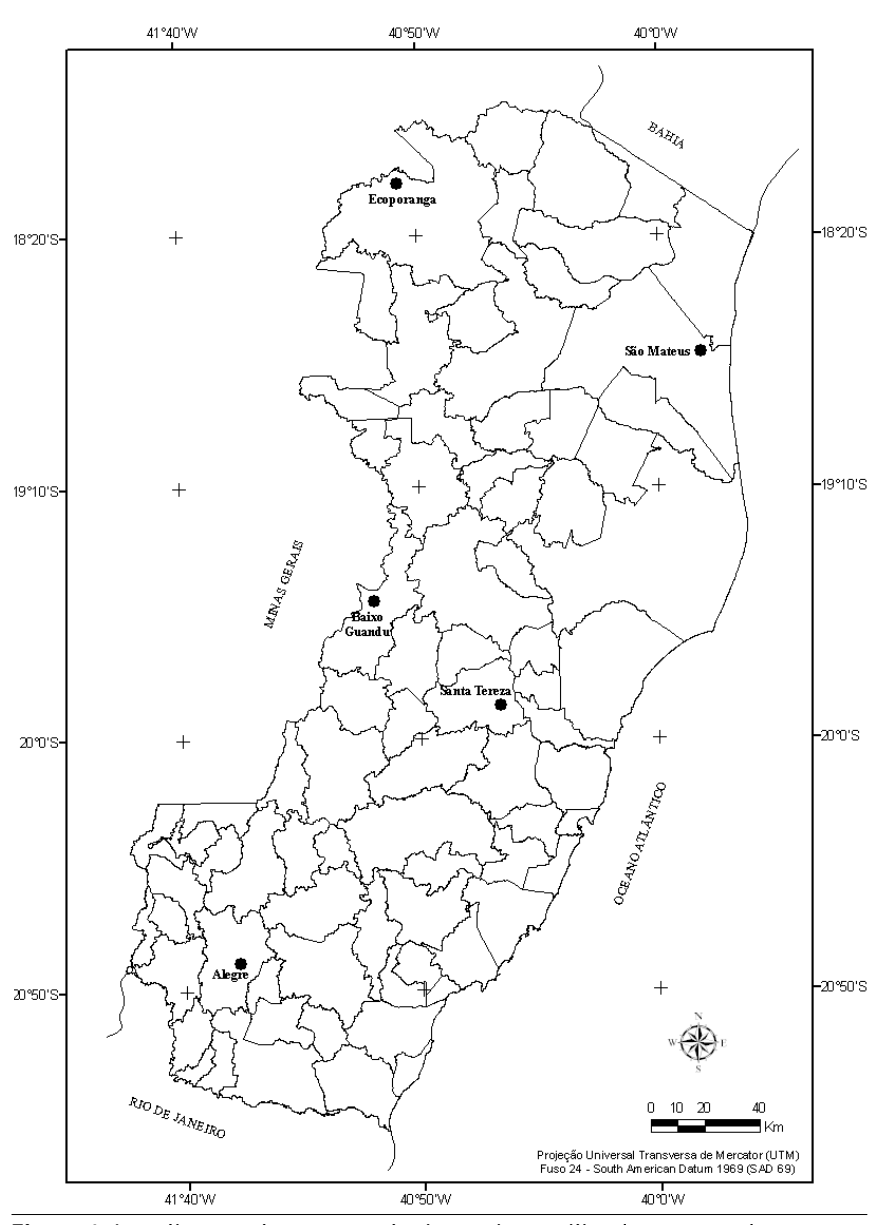

Figura 1. Localização dos postos pluviométricos utilizados no estudo

A caracterização do regime de precipitação pluvial foi realizada por meio do ajuste das séries empíricas à distribuição gama. A expressão da função densidade de probabilidade gama (FDPG) para uma variável x, é:

$$
\mathrm{g}(\mathrm{X})=\frac{\mathrm{X}^{\alpha-1} * \mathrm{e}^{-\mathrm{X} / \beta}}{\beta^{\alpha} \Gamma(\alpha)} \operatorname{para} \mathrm{X}>0
$$

em que:

a $>0$ - parâmetro de forma - adimensional

b > 0 - parâmetro de escala - mesma dimensão da variável $\mathrm{x}$

$\Gamma(\alpha)$ - a função gama

Calcularam-se os parâmetros $\alpha$ e $\beta$ pelo método da máxima verossimilhança, de acordo com a Eq. 2 e 3: 


$$
\begin{gathered}
\alpha=\frac{1}{4 \mathrm{~A}}(1+\sqrt{1+4 \mathrm{~A} / 3}) \ldots \beta=\overline{\mathrm{X}} / \alpha \\
\mathrm{A}=\ln (\overline{\mathrm{X}})-\sum(\ln (\mathrm{X})) / \mathrm{n}
\end{gathered}
$$

Conforme descrito em trabalhos como os de Blain et al. (2007) e Blain (2009), a função densidade probabilidade gama assume diversas formas, de conformidade com a variação de á: valores deste parâmetro inferiores a 1 resultam em uma distribuição fortemente assimétrica (forma exponencial) com $\mathrm{g}(\mathrm{x})$ tendendo ao infinito para $x$ tendendo a $0 . \operatorname{Em} \alpha=1$ a função intercepta o eixo vertical em $1 / \beta$ para $\mathrm{x}=0$. $\mathrm{O}$ aumento da magnitude deste parâmetro diminui o grau de assimetria (desvio em relação à moda) da distribuição (a densidade de probabilidade se desloca para a direita). Valores de $\alpha$ maiores que 1 resultam na FDPG com ponto máximo (moda) em $\beta^{*}(\alpha-1)$.

$\mathrm{O}$ aumento do parâmetro â alonga a FDPG para a direita, com redução de sua altura e diminuição da probabilidade de ocorrência do valor da moda. Analogamente e conforme a densidade seja comprimida para a esquerda (diminuição da magnitude de $\beta$ ) a altura da função se torna maior fazendo com que a probabilidade de ocorrência do valor da moda aumente.

A aderência das séries empíricas à distribuição paramétrica sob análise foi avaliada por meio dos testes KolmogorovSmirnov (KS) e qui-quadrado $\left(\chi^{2}\right)$ conforme descrito em Wilks (2006). Esses métodos adotam uma hipótese Ho que, quando não rejeitada, indica que a distribuição empírica pode ser considerada (no caso do presente estudo) oriunda de uma população com distribuição gama. Blain \& Brunini (2005) utilizaram esses dois testes a fim de verificar o ajuste das séries de precipitação pluvial, relativas às 12 localidades do Estado de São Paulo, a FDPG.

O nível de significância aqui adotado será $\alpha=10 \%$; ao contrário do KS, para a condução do teste $\chi^{2}$ é necessária a divisão dos dados em classes quantizadas; Neste sentido, Wilks (2006) afirma que, para variáveis contínuas, o teste KS é melhor aplicável quando comparado com o $\chi^{2}$.

Após a estimação de $\mathrm{g}(\mathrm{x})$ para as séries de precipitação sob investigação, o SPI pode ser calculado por:

$$
\begin{aligned}
& H(x)=q+(1-q) g(x) \\
& q=(m / n)
\end{aligned}
$$

em que:

q é a probabilidade de ocorrência de precipitação zero m é o número de observações com chuva igual a zero n é o número de observações com chuva maior que zero $\mathrm{H}(\mathrm{x})$ é, então, a transformada em uma variável normal (valor final do SPI) por meio da equação 8.

$$
\begin{gathered}
\mathrm{SPI}=-\left(\mathrm{a}-\frac{\mathrm{co}+\mathrm{c}_{1} \mathrm{t}+\mathrm{c}_{2} \mathrm{t}^{2}}{1+\mathrm{d}_{1} \mathrm{t}+\mathrm{d}_{2} \mathrm{t}^{2}+\mathrm{d}_{3} \mathrm{t}^{3}}\right) \text { para } 0<\mathrm{H}(\mathrm{x}) \leq 0,5 \\
\mathrm{SPI}=+\left(\mathrm{a}-\frac{\mathrm{co}+\mathrm{c}_{1} \mathrm{t}+\mathrm{c}_{2} \mathrm{t}^{2}}{1+\mathrm{d}_{1} \mathrm{t}+\mathrm{d}_{2} \mathrm{t}^{2}+\mathrm{d}_{3} \mathrm{t}^{3}}\right) \text { para } 0,5<\mathrm{H}(\mathrm{x})<0,5
\end{gathered}
$$

em que

$$
\begin{gathered}
\mathrm{a}=\sqrt{\left(\ln \left(\frac{1}{\mathrm{H}(\mathrm{x})^{2}}\right)\right)} \text { para } 0<\mathrm{H}(\mathrm{x}) \leq 0,5 \\
\mathrm{a}=\sqrt{\left(\ln \left(\frac{1}{\left(1-\mathrm{H}(\mathrm{x})^{2}\right)}\right)\right)} \text { para } 0,5<\mathrm{H}(\mathrm{x})<1 \\
\mathrm{c}_{0}=2,515517 ; \mathrm{c}_{1}=0,802853 ; \mathrm{c}_{2}=0,010328 ; \\
\mathrm{d}_{1}=1,432788 ; \mathrm{d}_{2}=0,189269 ; \mathrm{d}_{3}=0,0013
\end{gathered}
$$

O evento seca começa quando o SPI se torna negativo e atinge o valor de -1 e termina quando este volta a apresentar valores positivos. Dentro de sua escala, magnitudes menores ou iguais a -2 indicam seca extrema e maiores ou iguais a 2, umidade extrema, como se visualiza na Tabela 1.

Tabela 1. Valores do Índice Padronizado de Precipitação (SPI) e Categorias de seca ou de umidade

\begin{tabular}{cc}
\hline SPI & Categoria \\
$0-2,00$ & Extremamente Úmido \\
1,5 a 1,99 & Severamente Úmido \\
1,00 a 1,49 & Moderadamente Úmido \\
0.1 a 0,99 & Umidade Incipiente \\
0 a $-0,99$ & Seca Incipiente \\
$-1,00$ a-1,49 & Moderadamente Seco \\
$-1,50$ a $-1,99$ & Severamente Seco \\
$0-2,00$ & Extremamente Seco \\
\hline
\end{tabular}

Fonte: MacKee et al. (1993, 1995)

Segundo Hayes et al. (1999) valores do SPI com magnitudes menores que menos um tendem a ocorrer dezesseis vezes a cada cem anos, magnitudes do SPI menores que menos dois acontecem de duas a três vezes a cada cem anos e valores menores que menos três, apenas uma vez a cada duzentos anos. Cada valor de SPI corresponde a um único valor de chuva para um período de determinada escala de tempo (Mckee et al., 1993,; Hayes et al., 1999). Autores como Wu \& Kinter (2009), Quiring \& Kluver (2009) e Hwang \& Carbono (2009) utilizam o SPI na investigação da variabilidade espaço-temporal do regime de precipitação pluvial em diversas partes do globo.

A função autocorrelação (análise de persistência) pode ser definida como sendo a correlação entre os valores de uma mesma série, deslocados por um intervalo (lag). A estimação do coeficiente de autocorrelação $\left(\mathrm{r}_{\mathrm{k}}\right.$; que para o lag 0 apresentará sempre o valor 1) foi realizada conforme descrito em Wilks (2006). Usualmente, o limite de significância é estimado por meio do processo ruído branco. Valores de $r_{k}$ iguais ou inferiores ao do ruído branco, indicam que a série é livre de persistência (Wilks, 2006).

A variabilidade espacial das séries mensais do SPI foi inferida através da função correlação cruzada, conforme descrita em Wilks (2006). O coeficiente desta função $r_{x y(k)}$ representa a correlação entre duas séries $\mathrm{X}$ e $\mathrm{Y}$, em que $\mathrm{X}$ é deslocado de $\mathrm{K}$ observações (lags). No caso do presente estudo, os deslocamentos K possuem unidade de "mês" (lag = 1 representa 1 mês de deslocamento entre as séries $\mathrm{X}$ e Y, por exemplo). 


\section{RESULTADOS E DISCUSSÃO}

A aplicação dos testes $\chi^{2}$ e KS indica bom ajuste das séries mensais de precipitação à FDPG. As amostras mensais de todas as localidades podem ser consideradas oriundas de uma população com distribuição gama, conforme ilustrado na Tabela 2 .

Tabela 2. Valores dos testes Kolmogorav-Smirnov (KS) e quiquadrado $\left(\chi^{2}\right)$ relativos ao ajuste das séries mensais de precipitação pluvial (1977 a 2006) para as localidades de Baixo Guandu, Ecoporanga, Alegre, São Mateus e Santa Tereza, estado do Espírito Santo

\begin{tabular}{cccccc}
\hline Mês & BaixoGuandu & Ecoporanga & Alegre & São Mateus & Santa Tereza \\
Jan & 0,063 & 0,078 & 0,073 & 0,091 & 0,089 \\
Fev & 0,077 & 0,114 & 0,059 & 0,101 & 0,125 \\
Mar & 0,102 & 0,090 & 0,096 & 0,105 & 0,060 \\
Abr & 0,102 & 0,082 & 0,058 & 0,080 & 0,065 \\
Mai & 0,057 & 0,051 & 0,060 & 0,116 & 0,083 \\
Jun & 0,031 & 0,063 & 0,049 & 0,091 & 0,069 \\
Jul & 0,054 & 0,154 & 0,061 & 0,057 & 0,191 \\
Ago & 0,078 & 0,078 & 0,052 & 0,129 & 0,063 \\
Set & 0,093 & 0,078 & 0,119 & 0,080 & 0,054 \\
Out & 0,075 & 0,122 & 0,098 & 0,112 & 0,140 \\
Nov & 0,061 & 0,072 & 0,160 & 0,123 & 0,145 \\
Dez & 0,147 & 0,070 & 0,054 & 0,167 & 0,135 \\
\hline \multicolumn{5}{c}{ Teste $\chi^{2}$ entre parentes 0 valor crítico } & para $\alpha=10 \%$ \\
Jan & $6,70(14,68)$ & $5,29(14,68)$ & $6,26(14,68)$ & $5,39(14,68)$ & $6,88(14,68)$ \\
Fev & $5,94(14,68)$ & $7,88(14,68)$ & $6,08(14,68)$ & $7,88(14,68)$ & $6,08(14,68)$ \\
Mar & $10,83(12,02)$ & $11,32(12,02)$ & $10,78(12,02)$ & $11,07(12,02)$ & $10,87(12,02)$ \\
Abr & $8,32(12,02)$ & $9,21(12,02)$ & $6,14(12,02)$ & $6,21(12,02)$ & $6,34(12,02)$ \\
Mai & $7,92(10,64)$ & $8,39(10,64)$ & $5,53(10,64)$ & $7,39(10,64)$ & $7,53(10,64)$ \\
Jun & $8,40(10,64)$ & $7,58(10,64)$ & $6,12(10,64)$ & $8,01(10,64)$ & $7,22(10,64)$ \\
Jul & $6,28(10,64)$ & $4,06(10,64)$ & $6,98(10,64)$ & $4,32(10,64)$ & $5,98(10,64)$ \\
Ago & $7,77(10,64)$ & $6,52(10,64)$ & $6,67(10,64)$ & $6,72(10,64)$ & $5,67(10,64)$ \\
Set & $7,75(12,02)$ & $5,37(12,02)$ & $5,33(12,02)$ & $3,37(12,02)$ & $5,33(12,02)$ \\
Out & $5,40(12,02)$ & $5,68(12,02)$ & $5,39(12,02)$ & $3,68(12,02)$ & $3,31(12,02)$ \\
Nov & $3,70(14,68)$ & $4,37(14,68)$ & $5,68(14,68)$ & $5,37(14,68)$ & $6,08(14,68)$ \\
Dez & $7,95(14,68)$ & $4,93(14,68)$ & $5,51(14,68)$ & $4,73(14,68)$ & $5,51(14,68)$ \\
\hline
\end{tabular}

O ajuste das séries mensais de precipitação pluvial à distribuição gama possibilita o uso do SPI para quantificação/ monitoramento das condições de seca nas localidades do estudo, permitindo também que os parâmetros estimados da FDPG sejam usados na caracterização climática das séries mensais de precipitação pluvial utilizadas neste trabalho.

Nas Tabelas 3 e 4 são ilustrados, respectivamente, os parâmetros $\alpha$ e $\beta$ das FDPG mensais, a média (M) e o coeficiente de variação (CV\%) de cada agrupamento mensal.

Nota-se, por meio das Tabelas 3 e 4 que, de forma geral, o período entre maio e setembro é caracterizado por pequenos totais mensais de precipitação pluvial, época do ano em que baixos teores de umidade do solo são previstos. No mês de outubro se observa aumento no regime de precipitação pluvial nas localidades do estudo; entretanto, neste mês ainda se observa baixos valores do parâmetro á e, consequentemente, altos valores de CV\%, indicando possibilidades significati-
Tabela 3. Parâmetros da distribuição gama relativos às séries mensais de precipitação pluvial (1977 a 2006) para as localidades de Baixo Guandu, Ecoporanga, Alegre, São Mateus e Santa Tereza do estado do Espírito Santo

\begin{tabular}{|c|c|c|c|c|c|c|c|c|c|c|}
\hline \multirow{2}{*}{ Mês } & \multicolumn{2}{|c|}{ BaixoGuandu } & \multicolumn{2}{|c|}{ Ecoporanga } & \multicolumn{2}{|c|}{ Alegre } & \multicolumn{2}{|c|}{ São Mateus } & \multicolumn{2}{|c|}{ SantaTereza } \\
\hline & $\alpha$ & $\beta$ & $\alpha$ & $\beta$ & $\alpha$ & $\beta$ & $\alpha$ & $\beta$ & $\alpha$ & $\beta$ \\
\hline 1 & 1,9 & 77,3 & 1,5 & 104,0 & 3,0 & 69,1 & 1,3 & 118,3 & 2,2 & 102,6 \\
\hline 2 & 1,1 & 67,5 & 2,3 & 45,4 & 1,5 & 74,4 & 1,3 & 60,3 & 2,1 & 56,0 \\
\hline 3 & 1,7 & 60,8 & 2,6 & 48,3 & 2,6 & 61,5 & 1,7 & 83,1 & 1,8 & 96,3 \\
\hline 4 & 1,2 & 39,9 & 3,1 & 23,7 & 2,1 & 54,4 & 2,7 & 38,7 & 5,1 & 18,0 \\
\hline 5 & 1,1 & 30,3 & 2,2 & 17,9 & 3,6 & 14,9 & 1,4 & 43,1 & 1,3 & 44,6 \\
\hline 6 & 0,9 & 11,0 & 1,2 & 23,9 & 1,3 & 18,2 & 1,3 & 44,6 & 1,3 & 34,2 \\
\hline 7 & 0,8 & 13,1 & 1,2 & 27,6 & 1,3 & 22,0 & 1,2 & 51,8 & 1,5 & 36,6 \\
\hline 8 & 0,9 & 20,9 & 1,3 & 22,3 & 1,2 & 31,0 & 1,7 & 29,7 & 1,5 & 41,5 \\
\hline 9 & 0,9 & 28,7 & 1,4 & 25,3 & 1,7 & 39,2 & 1,3 & 63,4 & 1,6 & 41,5 \\
\hline 10 & 1,9 & 38,7 & 2,5 & 39,9 & 5,4 & 20,5 & 2,3 & 54,5 & 2,4 & 46,1 \\
\hline 11 & 2,8 & 56,2 & 3,7 & 45,9 & 5,9 & 38,5 & 2,8 & 73,4 & 3,0 & 77,6 \\
\hline 12 & 6,2 & 31,0 & 4,9 & 39,9 & 6,2 & 39,9 & 5,0 & 35,2 & 5,5 & 44,0 \\
\hline
\end{tabular}

Tabela 4. Média (M) e coeficiente de variação (CV\%) relativo às séries mensais de precipitação pluvial (1977 a 2006) para as localidades de Baixo Guandu, Ecoporanga, Alegre, São Mateus e Santa Tereza do estado do Espírito Santo

\begin{tabular}{|c|c|c|c|c|c|c|c|c|c|c|}
\hline \multirow{2}{*}{ Mês } & \multicolumn{2}{|c|}{ BaixoGuandu } & \multicolumn{2}{|c|}{ Ecoporanga } & \multicolumn{2}{|c|}{ Alegre } & \multicolumn{2}{|c|}{ SãoMateus } & \multicolumn{2}{|c|}{ SantaTereza } \\
\hline & $M$ & CV\% & $M$ & CV\% & $M$ & $\mathrm{CV} \%$ & $M$ & CV\% & $M$ & CV\% \\
\hline 1 & 150,6 & 71,7 & 154,9 & 81,9 & 208,6 & 57,6 & 156,8 & 86,9 & 226,8 & 67,3 \\
\hline 2 & 71,4 & 97,2 & 102,7 & 66,5 & 113,6 & 80,9 & 81,2 & 86,2 & 116,9 & 69,2 \\
\hline 3 & 103,0 & 76,8 & 127,5 & 61,5 & 160,5 & 61,9 & 141,9 & 76,5 & 169,1 & 75,5 \\
\hline 4 & 47,5 & 91,7 & 74,6 & 56,4 & 114,1 & 69,1 & 105,0 & 60,7 & 2,5 & 44,1 \\
\hline 5 & 34,5 & 93,7 & 39,3 & 67,4 & 54,1 & 52,5 & 59,6 & 85,0 & 56,6 & 88,8 \\
\hline 6 & 10,2 & 104,0 & 28,2 & 92,0 & 23,1 & 88,7 & 56,2 & 89,1 & 44,6 & 87,6 \\
\hline 7 & 11,0 & 109,0 & 32,6 & 92,0 & 27,5 & 89,3 & 64,5 & 89,6 & 56,0 & 80,9 \\
\hline 8 & 18,3 & 106,7 & 29,6 & 86,8 & 36,6 & 92,0 & 51,7 & 75,8 & 63,4 & 80,9 \\
\hline 9 & 25,1 & 107,0 & 35,2 & 84,8 & 65,5 & 77,4 & 83,9 & 87,0 & 66,7 & 78,9 \\
\hline 10 & 73,7 & 72,4 & 98,5 & 63,6 & 111,0 & 43,0 & 122,8 & 66,6 & 112,5 & 64,0 \\
\hline 11 & 158,7 & 59,5 & 170,0 & 52,0 & 228,6 & 41,0 & 202,8 & 60,2 & 230,9 & 58,0 \\
\hline 12 & 191,8 & 40,2 & 196,2 & 45,1 & 249,0 & 40,0 & 176,0 & 44,7 & 242,9 & 42,6 \\
\hline
\end{tabular}

vas de ocorrência de baixos totais mensais de precipitação pluvial; desta forma, as atividades de semeadura/plantio no mês de outubro envolvem considerável grau de risco aos danos causados por estresse hídrico. A localidade do Baixo Guandu é a que apresenta o maior potencial de ocorrência de baixos valores de precipitação no mês de outubro.

Pode-se indicar, entre os meses de novembro e março, que a estação chuvosa da região está climatologicamente estabelecida. Em virtude dos maiores valores do parâmetro á e dos menores valores de $\mathrm{CV} \%$, os meses de novembro e dezembro são, de forma geral, os que apresentam os menores riscos de ocorrência de seca agrícola; tal risco, por outro lado, se torna maior nos meses de janeiro a março devido ao maior potencial de ocorrência de veranicos nas localidades estudadas, principalmente nos municípios de Baixo Guandu e São Mateus, que apontam os menores valores do parâmetro á para o período; já o mês de abril pode ser visto como mês de transição entre as estações seca e chuvosa da região. De forma geral, as localidades de Alegre e Santa Tereza apresentam os meno- 


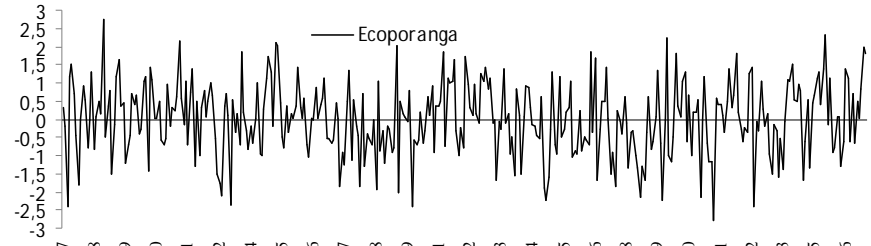

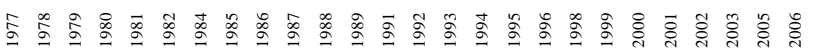
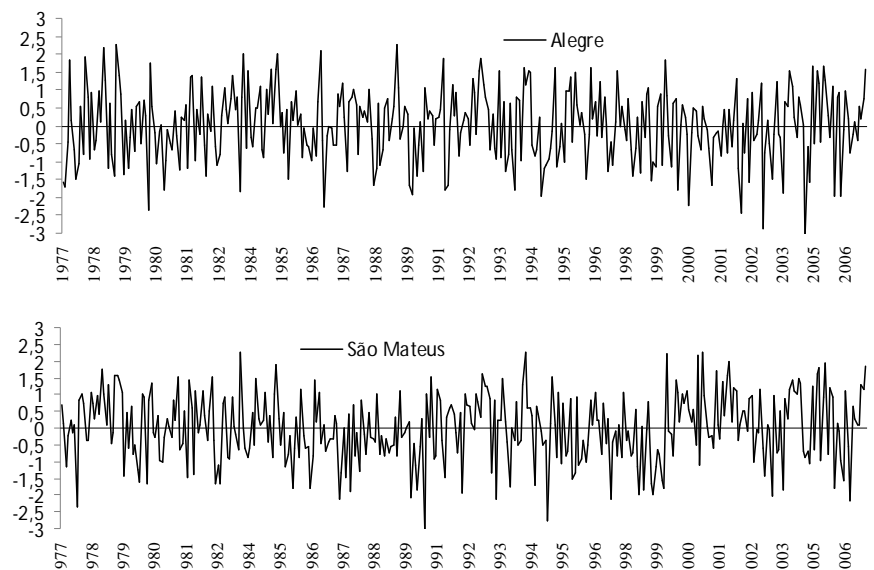

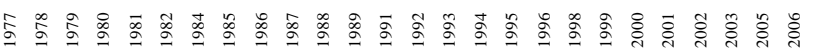

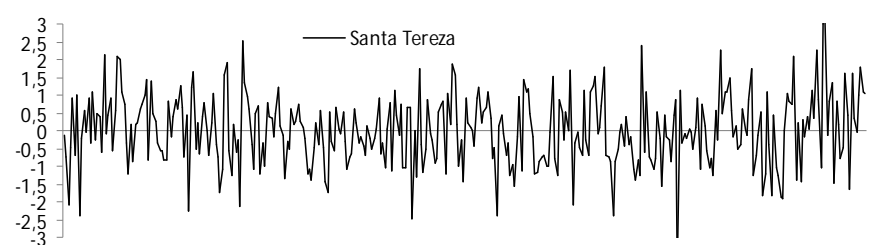

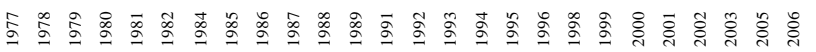

Figura 2. Índice Padronizado de Precipitação (SPI), na escala mensal, relativos às localidades de Baixo Guandu, Ecoporanga, Alegre, São M ateus e Santa Tereza, Estado do Espírito Santo

res potenciais de ocorrência do fenômeno da seca agrícola entre as localidades analisadas.

Após o ajuste das séries mensais de precipitação à distribuição gama, empregou-se o SPI para o monitoramento das condições de seca. É ilustrada, na Figura 2, a variação temporal do SPI na escala mensal para as localidades de Baixo Guandu, Ecoporanga, Alegre, São Mateus e Santa Tereza, entre os anos de 1971 a 2006. É interessante ressaltar que as localidades de Alegre, Ecoporanga, São Mateus e Santa Tereza, apresentaram frequência de ocorrência das classes do SPI bastante próximas à citada por Hayes et al. (1999), com aproximadamente $68 \%$ dos valores compreendidos entre -1 e 1; $95 \%$ entre -2 e 2 e $99 \%$ entre -3 e 3 . A localidade de Baixo Guandu indicou $75 \%$ dos valores compreendidos entre $-1 \mathrm{e}$ 1; $96 \%$ entre -2 e 2 e $100 \%$ entre -3 e 3 (condições mais extremas de déficit e excesso de precipitação ocorrem com menor frequência).

Outro aspecto a ser observado na Figura 2, é a grande variabilidade espaço-temporal inerente ao regime de precipitação pluvial do Estado do Espírito Santo. Em uma mesma região meses extremamente secos podem ser precedidos e seguidos de meses úmidos ou normais como, por exemplo, pode ser citado julho de 1990, na localidade de São Mateus (SPI $=-3,1)$ ao passo que junho e agosto do mesmo ano apresentaram os valores 0,3 e 1 , respectivamente, característica que indica que as afirmações de Melo Júnior et al. (2006), relativas ao caráter aleatório da precipitação pluvial, também podem ser observadas nas séries mensais do SPI, ou seja, nas condições de seca meteorológica das cinco localidades do estudo. Esta característica é corroborada pela análise de persistência apresentada na Figura 3, para as cinco localidades.

Nota-se, na Figura 3 que, durante o período analisado as localidades de Ecoporanga, Santa Tereza e Baixo Guandu apresentaram valores positivos e significativos de $r_{k}$ no lag 1 , indicando que nesses municípios determinada condição hídrica (déficit ou excesso de precipitação) teve, em relação às demais localidades, maiores possibilidades de perdurar até o mês subsequente. O caráter aleatório dos valores do SPI é bastante evidente no município de Alegre, em que foi registrado valor positivo e significativo de $\mathrm{r}_{\mathrm{k}}$ no lag 6 , ao passo que no lag 24 (período relativo a 2 anos) se registrou valor negativo e significativo de $\mathrm{r}_{\mathrm{k} \text {. }}$; neste sentido, é interessante ressaltar que no município de São Mateus foram registrados
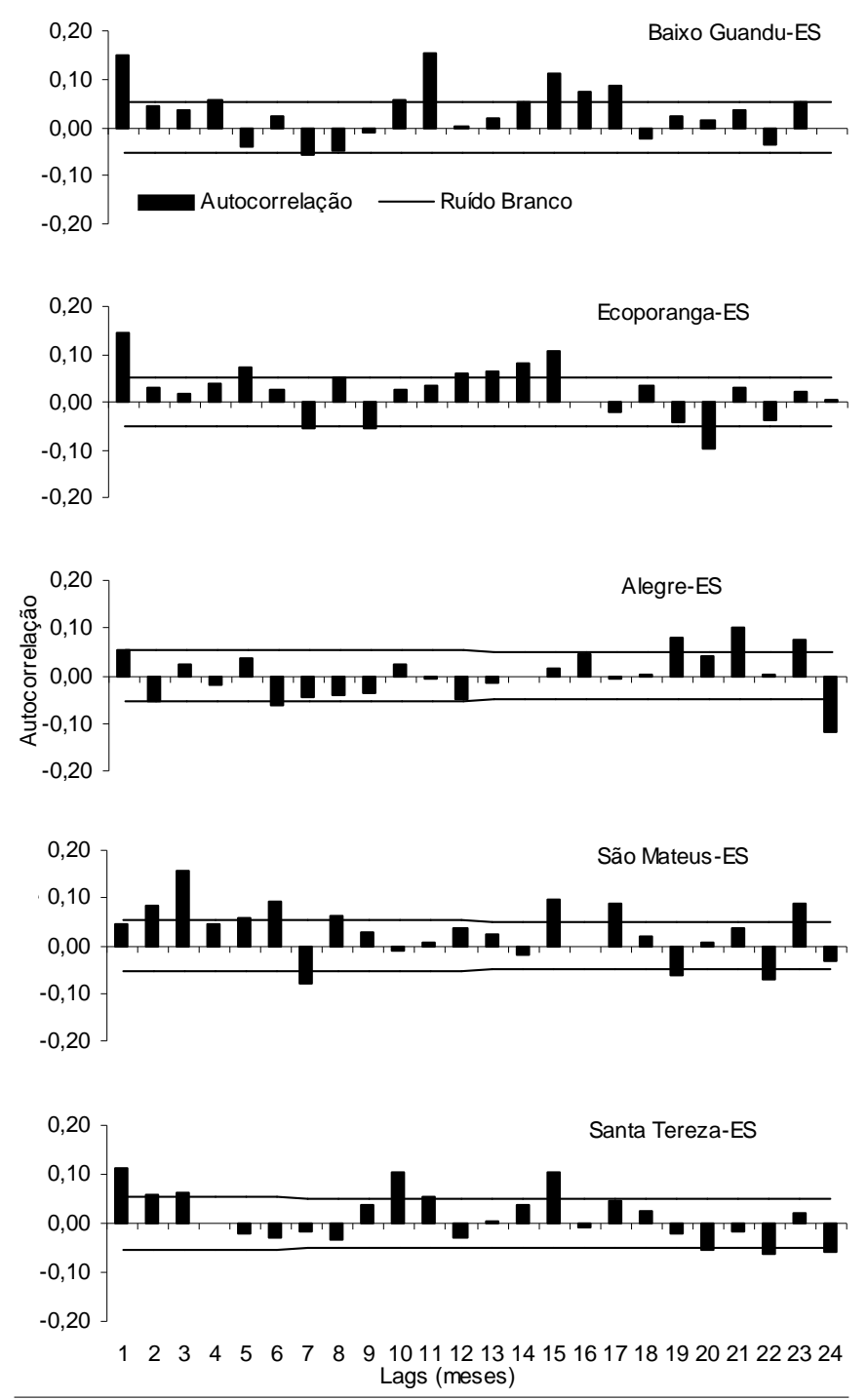

Figura 3. Coeficientes da função autocorrelação, lags 1 a 24 (meses), para as localidades Baixo Guandu, Ecoporanga, Alegre, São M ateus e Santa Tereza, Estado do Espírito Santo 
valores positivos e significativos de $r_{k}$ nos lags 5 e 6 , enquanto o lag 7 apresentou valores negativos e significativos de $r_{k}$.

Constatou-se, então, que a principal característica da dinâmica temporal das séries do SPI mensal, nas cinco localidades, é o caráter aleatório em que uma condição de déficit (ou excesso) de precipitação pluvial pode ter início ou fim de forma bastante abrupta; neste sentido, é oportuno ressaltar as afirmações de Lima et al. (2008) que, ao avaliarem a variabilidade temporal dos valores mensais de precipitação pluvial na localidade de Alegre constataram, por meio da construção de semivariogramas, o chamando efeito pepita puro, ou seja, ausência de dependência temporal caracterizando variabilidade aleatória nos dados desse elemento meteorológico.

Considerando que os coeficientes $r_{k}$ foram aplicados em um período homogêneo nas cinco localidades, os baixos, porém distintos, graus de persistência das séries mensais de SPI podem indicar, além da grande variabilidade temporal, elevada variabilidade espacial dos valores desse índice de seca; em outras palavras, durante um mesmo mês as cinco localidades podem apresentar condições de seca bastante diferentes; esta inferência é corroborada pelos resultados apresentados na Tabela 5 relativos à aplicação da função correlação cruzada.

Pela Tabela 5 e Figura 3, nota-se grande variabilidade espacial dos valores do SPI mensal (e, consequentemente, do regime de precipitação) das localidades do Espírito Santo. Considerando-se o lag 0, as localidades de Baixo Guandu e Santa Tereza, e Ecoporanga e São Mateus, são as que apresentam as maiores correlações entre as séries do SPI. A forte queda em todas as localidades do valor de $r_{x y(k)}$ para lags diferentes de 0 é um forte indicativo da grande variabilidade espacial das distintas condições de seca que podem ocorrer no Estado do Espírito Santo.

Tabela 5. Coeficiente da função correlação cruzada $\left(r_{x y(k)}\right)$ para as localidades de Baixo Guandu, Ecoporanga, Alegre, São Mateus e Santa Tereza, considerados os deslocamentos (L) -3 a 3 meses

\begin{tabular}{cccccc}
\hline \multicolumn{6}{c}{ Coeficiente da função correlação cruzada (ruído branco = 0,052) } \\
\hline & $\begin{array}{c}\text { Baixo } \\
\text { Guandu }\end{array}$ & $\begin{array}{c}\text { Baixo } \\
\text { Guandú }\end{array}$ & $\begin{array}{c}\text { Baixo } \\
\text { Guandu }\end{array}$ & $\begin{array}{c}\text { Baixo } \\
\text { Guandú }\end{array}$ & Alegre \\
\cline { 2 - 6 } & Ecoporanga & Alegre & São Mateus & $\begin{array}{c}\text { Santa } \\
\text { Tereza }\end{array}$ & $\begin{array}{c}\text { Santa } \\
\text { Tereza }\end{array}$ \\
-3 & 0,054 & $-0,025$ & 0,013 & 0,097 & $-0,043$ \\
-2 & 0,049 & $-0,010$ & 0,081 & 0,071 & 0,032 \\
-1 & 0,193 & 0,139 & 0,140 & 0,111 & 0,077 \\
0 & 0,588 & 0,532 & 0,445 & 0,605 & 0,497 \\
1 & 0,103 & 0,062 & 0,037 & 0,141 & 0,071 \\
2 & 0,022 & 0,035 & $-0,002$ & 0,037 & $-0,015$ \\
3 & 0,043 & 0,032 & 0,017 & $-0,035$ & 0,071 \\
\hline \multirow{6}{*}{ L } & Ecoporanga & Ecoporanga & Ecoporanga & Alegre & São Mateus \\
\cline { 2 - 6 } & Alegre & São Mateus & Santa Tereza São Mateus & Santa Tereza \\
-3 & 0,001 & 0,029 & $-0,012$ & 0,066 & 0,080 \\
-2 & 0,056 & 0,033 & 0,027 & 0,021 & 0,002 \\
-1 & 0,148 & 0,104 & 0,072 & 0,122 & 0,011 \\
0 & 0,400 & 0,652 & 0,587 & 0,358 & 0,550 \\
1 & 0,052 & 0,074 & 0,162 & 0,025 & 0,125 \\
2 & $-0,071$ & 0,036 & 0,010 & $-0,059$ & 0,025 \\
3 & $-0,003$ & 0,037 & 0,084 & 0,007 & 0,128 \\
\hline
\end{tabular}

\section{CONCLUSÕES}

1. As séries mensais de precipitação pluvial das localidades de Baixo Guandu, Ecoporanga, Alegre, São Mateus e Santa Tereza, pertencentes ao Estado do Espírito Santo, podem ser consideradas oriundas de uma população com distribuição gama incompleta permitindo, com isto, o uso do Índice Padronizado de Precipitação (SPI) no monitoramento das condições de seca.

2. A principal característica das séries do SPI é sua grande variabilidade espaço-temporal, a qual indica que, em uma mesma região, meses extremamente secos podem ser precedidos e seguidos de meses úmidos ou normais, e distintos casos de seca podem ocorrer de forma aleatória entre as localidades, em um mesmo período.

\section{LITERATURA CITADA}

Blain, G. C. Considerações estatísticas relativas a oito séries de precipitação pluvial da Secretaria de Agricultura e Abastecimento do Estado de São Paulo, Revista Brasileira de Meteorologia, v.24, n.1 p.12-23, 2009.

Blain, G. C.; Brunini, O. Avaliação e adaptação do Índice de Severidade de Seca de Palmer (PDSI) e do Índice Padronizado de Precipitação (SPI) às condições climáticas do Estado de São Paulo. Bragantia, v.64, n.4, p.695-705, 2005.

Blain, G. C.; Piedade, S. M. S.; Camargo, M. B. P.; Giarola, A. Distribuição temporal da precipitação pluvial mensal observada no posto meteorológico do Instituto Agronômico, em Campinas, SP. Bragantia, v.66, n.2, p.347-355, 2007.

Hayes, M. J.; Svoboda, M. D.; Wilhite, D. A.; Vanyarkho, O. V. Monitoring the 1996 drought using the Standardized Precipitation Index. Bulletin of the American Meteorological Society, v.80, n.3, p.429-438, 1999.

Hwang, Y.; Carbone, G. J. Ensemble Forecasts of Drought Indices Using a Conditional Residual Resampling Technique. Journal of Applied Meteorology and Climatology, v.48, n.7, p.1289-1301, 2009.

Lima, J. S. S.; Silva, S. A.; Oliveira, R. B.; Cecílio, R. A.; Xavier, A. C. Variabilidade temporal da precipitação mensal em Alegre ES. Revista Ciência Agronômica, v.39, n.2, p. 327-332, 2008

McKee,T. B.; Doesken, N. J.; Kleist, J. The relationship of drought frequency and duration to times scale. In: Conference on Appied Climatology, 8.,1993, Boston. Anais American Meteorological Society. Boston: Preprints, 1993. p.179-184.

Melo Júnior, J. C. F.; Seditama, G. C.; Ferreira, P. A.; Brauliro, G. L. Distribuição espacial da frequência de chuvas na região hidrográfica do Atlântico, Leste de Minas Gerais. Revista Brasileira de Engenharia Agrícola e Ambiental, v.10, n.2, p.417-425, 2006.

Quiring, S. M.; Kluver, D. B. Relationship between winter/spring snowfall and summer precipitation in the Northern Great Plains of North America. Journal of Hydrometeorology, v.10, n.5, p.1207-1207, 2009.

Silva, J. W.; Guimarães, E. C.; Tavares, M. Variabilidade temporal da precipitação mensal e anual na estação climatológica de Uberaba-MG. Ciência e Agrotecnologia, v.27, n.3, p. 665-674, 2003. 
Vieira, D. B.; Lombardi Neto, F.; Santos, R. P. Análise das intensidades máximas de chuva em Pindorama (SP). Revista Brasileira de Ciência do Solo, v.18, p.255-260, 1994.

Wilhite, D. A.; Glantz, M. H. Understanding the drought phenomenon: The role definations. In: Wilhite D. A.; Easterling, W. E.; Wood, D. W. Planning for drought toward a reduction of societal vulnerability. Boulder: Westview Press, 1987. p.11-14.
Wilks, D. S. Theoretical probability distributions. In: Statistical methods in the atmospheric sciences. 2.ed. San Diego: Academic Press, 2006. 627p.

Wu, R.; Kinter, L. J. Analysis of the Relationship of U.S. Droughts with SST and Soil moisture: Distinguishing the time scale of droughts. Journal of Climate, v.22, n.17, p.45204538, 2009. 